\title{
Improved Indoor Localization Based on Received Signal Strength Indicator and General Regression Neural Network
}

\author{
Shuqi Xu, ${ }^{1}$ Zhuping Wang, ${ }^{1 *}$ Hao Zhang, ${ }^{\text {*** }}$ and Shuzhi Sam $\mathrm{Ge}^{2,3}$ \\ ${ }^{1}$ College of Electronics and Information Engineering, Tongji University, \\ No. 4800 Caoan Road, Jiading District, Shanghai 201804, China \\ ${ }^{2}$ Department of Electrical \& Computer Engineering, National University of Singapore, \\ 21 Lower Kent Ridge Road, Singapore 117576, Singapore \\ ${ }^{3}$ Institute For the Future (IFF), Qingdao University, \\ No. 308 Ningxia Road, Qingdao, Shandong 266071, China
}

(Received January 6, 2019; accepted April 15, 2019)

Keywords: received signal strength indicator (RSSI), ZigBee, localization, filter, maximum likelihood estimation (MLE), general regression neural network (GRNN)

Nowadays, indoor positioning is becoming one of the most important issues in smart cities. With the rapid progress of wireless communication and digital electronic technology, wireless sensor networks (WSNs) have been developed and are playing an important role in indoor positioning systems. The received signal strength indicator (RSSI) is adopted by most rangebased localization algorithms. However, the positioning system based on the RSSI is vulnerable to environmental interference and the RSS itself is unstable. To tackle this problem, we propose an improved indoor localization based on the RSSI and general regression neural network (GRNN). In the raw data processing module, an improved average filter is proposed to make the raw data stable and reliable. Then, an improved weighted centroid localization algorithm (IWCLA) is proposed to revise the positioning result on the basis of maximum likelihood estimation (MLE). In the view of the complex and changeable indoor environment, an improved GRNN localization algorithm is proposed to achieve better applicability and higher positioning accuracy. The effectiveness of the proposed methods is verified in different cases through simulation and experiment studies.

\section{Introduction}

The construction of smart cities is a developing trend throughout the world. ${ }^{(1,2)}$ The cities should be people-oriented and should achieve comprehensive and sustainable economic, social, and environmental developments. The development of positioning technology has become an indispensable part of the construction of smart cities. It is widely used in various fields, such as public safety, social networks, intelligent transportation, safe medical treatment, and location tracking. Positioning technology is divided into indoor and outdoor positioning. The

\footnotetext{
*Corresponding author: e-mail: elewzp@tongji.edu.cn

** Corresponding author: e-mail: zhang_hao@tongji.edu.cn https://doi.org/10.18494/SAM.2019.2253
} 
global positioning system (GPS) ${ }^{(3)}$ and global navigation satellite system (GNSS) ${ }^{(4)}$ both need to be maintained with the direct vision of the satellite in the outdoor environment in order to achieve the quality of communication applications. Therefore, the GPS and GNSS are efficient as outdoor positioning systems, but not indoor positioning systems. Indoor positioning is more difficult. The complexity of the indoor positioning environment, the complex and changeable indoor space layout, multiple obstacles, multiple sources of interference, and other factors have great impacts on the accuracy and stability of indoor positioning. The study of indoor positioning systems is essential. ${ }^{(5,6)}$

Indoor positioning, a research hotspot, is widely used in applications such as vehicle positioning. $\mathrm{Qu}$ et al. used the technology of visual odometry to achieve a positioning accuracy of higher than $5 \mathrm{~m} .{ }^{(7)}$ Wagner et al. used the technology of visual odometry and a gyro to achieve a positioning accuracy of higher than $2.5 \mathrm{~m} .{ }^{(8)}$ Ibisch et al. used multicamera technology to achieve a positioning accuracy of higher than $1.2 \mathrm{~m} .{ }^{(9)}$ These methods are all vision-related and require the target to be in sight. In a real environment, such as a garage, there may exist disturbances or occlusions due to the large number of objects in the complex environment. The indoor positioning methods mentioned above have certain limitations, and hence, a method based on the wireless sensor network (WSN) and received signal strength indicator (RSSI) is more applicable. ${ }^{(10,11)}$ Lin et al. used an optimized fingerprint-based positioning algorithm to achieve a positioning accuracy of higher than $3.5 \mathrm{~m}$ in a garage. ${ }^{(12)}$ In this work, we optimize the general radio-propagation-model-based localization algorithm to achieve an accuracy of higher than $4 \mathrm{~m}$. On the other hand, an improved general regression neural network (GRNN) positioning method based on an optimized method is proposed to make the positioning accuracy higher than $2.4 \mathrm{~m}$.

At present, the indoor localization technology based on the WSN and RSSI mainly includes WIFI, ${ }^{(13)}$ Bluetooth, ${ }^{(14)}$ ZigBee, ${ }^{(15)}$ and so forth. The accuracy of WIFI localization can only reach about $2 \mathrm{~m}$. The stability of Bluetooth localization is low owing to signal interference, and Bluetooth devices are relatively expensive. ZigBee localization has the advantages of low cost, reliable data transmission, stable network, low power consumption, and simple network layout. It supports a large number of nodes by using many fixed nodes to detect the RSSI value with an unknown destination node. Then, better nodes can be chosen to estimate the unknown position of the blind node. An indoor positioning experiment is performed using ZigBee to verify our methods.

In 2001, Laitinen et al. were the first to propose the location fingerprint-based positioning technology, namely, the database correlation method, which achieved significantly good positioning performance. ${ }^{(16)}$ Since the positioning performance mainly depends on the size and quality of the fingerprint database and the process of collecting data is time-consuming, the main challenges of the fingerprint-based positioning technology lie in two aspects. The first is to increase the positioning accuracy, and the second is to reduce the workload. ${ }^{(17-21)}$

With the fingerprint database, intelligent algorithms especially neural network (NN) algorithms can be applied to achieve a high positioning accuracy. The back-propagation neural network (BPNN) is in common use, ${ }^{(22,23)}$ but has a local minimum problem. ${ }^{(24)}$ The radial basis function neural network (RBFNN) has been developed to solve this problem. ${ }^{(25,26)}$ However, 
it encounters difficulties in the determination of the number and center of hidden layer nodes in the network. The GRNN is a regularized RBFNN that overcomes the disadvantages of the RBFNN to some extent. ${ }^{(27)}$ When the number of input samples is large enough, the network can approximate a function with arbitrary precision. However, with the increase in the number of samples, the network becomes larger and the computational efficiency decreases. Thus, proper dimensionality reduction is needed. In this paper, improved indoor localization methods based on the RSSI and GRNN are proposed. The main contributions are listed as follows.

(1) An improved radio-propagation-model-based localization algorithm is proposed. In the raw data processing module, an improved average filter is proposed to make the raw data stable and reliable. Then, the improved weighted centroid localization algorithm (IWCLA) is proposed to revise the positioning result by maximum likelihood estimation.

(2) An improved GRNN-based localization method is proposed to increase the applicability and accuracy of the positioning system in the real environment.

The rest of this paper is organized as follows. In Sect. 3, an improved filtering algorithm is proposed. In Sect. 4, the use of maximum likelihood estimation (MLE) in the positioning problem and the revision of the position of the nodes to be tested by the IWCLA are described. Section 5 shows a description of the improved GRNN. The simulation and experiment results are given in Sects. 6 and 7. In Sect. 8, we present our conclusions.

\section{Radio-propagation Path Loss Model}

For signals propagating from the transmitter to the receiver, varying degrees of loss exist. To a large extent, the loss affects the accuracy of positioning based on the RSSI, so selecting an appropriate loss model is particularly important.

The two theoretical models most commonly used are the free space propagation loss model (free space) used in the ideal environment and the lognormal distribution model (shadowing) used in the actual environment. ${ }^{(28)}$

Signal interference has a serious impact on signal intensity loss. ${ }^{(29)}$ In this case, the free space propagation loss model is not applicable. Therefore, the shadowing model (SM) is introduced. The relationship between the received power and the distance is given by

$$
P L(d)=P L\left(d_{0}\right)+10 \times n \times \lg \left(d / d_{0}\right)+X_{\sigma},
$$

where $d$ is the distance between the receiver and the transmitter, $d_{0}$ is the reference distance, $n$ is known as the path loss exponent, $P L(d)$ is the signal power $(\mathrm{dBm})$ of the receiver, and $X_{\sigma}$ denotes a zero mean Gaussian random variable that reflects the interference in an indoor environment.

In a practical application, it is desirable that $d_{0}=1 \mathrm{~m}$ and that the RSSI values of the receiver can be collected. Therefore, the simple model can be represented as

$$
P L(d)=A+B \times \lg (d),
$$


where $A=P L(1)+X_{\sigma}$ and $B=10 \times n$. From the known data of the received power and distance, the values of $\mathrm{A}$ and $\mathrm{B}$ can be calculated by linear fitting.

\section{Improved Average Filter for RSSI}

In the indoor wireless network, the multipath and shadow effects can easily cause the fluctuation of the RSSI value. In addition, owing to the complex and changeable indoor environment and frequent activities of indoor personnel, the received value of the RSSI will fluctuate around the real values. The use of a filter to remove the noise is necessary. Various filters can be used to smooth the RSSI value. The simplest one is the average filter. ${ }^{(30)}$

The average RSSI value requires a few packets from each beacon node. The RSSI value is calculated as

$$
\overline{R S S I}=\frac{1}{n} \sum_{i=1}^{n} R S S I_{i}
$$

To better remove the sudden peaks, we adopt a new filter based on the average filter. The filtered value $\widehat{R S S I}$ is given by Eq. (4) by assigning each weight.

$$
\widetilde{R S S I}=\sum_{i=1}^{n}\left(1-\frac{\left|R S S I_{i}-\overline{R S S I}\right|^{m}}{\sum_{i=1}^{n}\left|R S S I_{i}-\overline{R S S I}\right|^{m}}\right) \times \frac{R S S I_{i}}{n-1}
$$

\section{Radio-propagation-model-based Localization Algorithm}

Since the original positioning system based on the RSSI is vulnerable to environmental interference and the RSS itself is unstable, the positioning accuracy becomes low. In order to improve the positioning accuracy, the general positioning algorithm is optimized. The positioning module can be divided into three phases. First, the number of nearest beacon nodes for the blind node is determined in the determination phase. Then, the initial positioning result of the blind node is calculated using the selected nearest beacon nodes in the positioning phase. In the correction phase, the initial positioning result is fine-tuned to obtain more accurate positioning coordinates.

\subsection{Maximum likelihood estimation}

MLE is a statistical method. ${ }^{(31)}$ As shown in Fig. 1, the known coordinates of the beacon nodes are $\left(x_{1}, y_{1}\right),\left(x_{2}, y_{2}\right),\left(x_{3}, y_{3}\right), \ldots,\left(x_{n}, y_{n}\right)$, and the distances between the beacon nodes and the blind node are $d_{1}, d_{2}, d_{3}, \ldots, d_{n}$, respectively, assuming that the coordinates of the blind node are $(X, Y)$. 


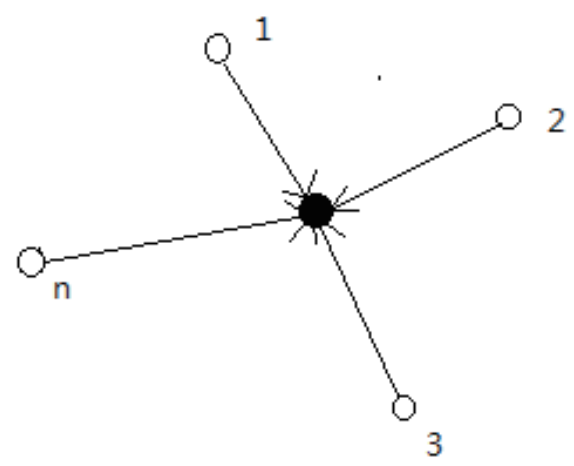

Fig. 1. Maximum likelihood estimation.

Then, Eq. (5) is established as

$$
\left\{\begin{array}{l}
\left(x_{1}-X\right)^{2}+\left(y_{1}-Y\right)^{2}=d_{1}^{2} . \\
\left(x_{n}-X\right)^{2}+\left(y_{n}-Y\right)^{2}=d_{n}^{2} .
\end{array}\right.
$$

The equations are changed into the form of $A Z=b$, such as

$$
A=\left[\begin{array}{cc}
2\left(x_{1}-x_{n}\right) & 2\left(y_{1}-y_{n}\right) \\
\ldots & \ldots \\
2\left(x_{n-1}-x_{n}\right) & 2\left(y_{n-1}-y_{n}\right)
\end{array}\right], b=\left[\begin{array}{c}
x_{1}^{2}-x_{n}^{2}+y_{1}^{2}-y_{n}^{2}+d_{n}^{2}-d_{1}^{2} \\
x_{n-1}^{2}-x_{n}^{2}+y_{n-1}^{2}-y_{n}^{2}+d_{n}^{2}-d_{n-1}^{2}
\end{array}\right], z=\left[\begin{array}{c}
X \\
Y
\end{array}\right]
$$

Utilizing the standard minimum variance estimation method, the coordinate of the blind node can be obtained as

$$
Z=\left(A^{\mathrm{T}} A\right)^{-1} A^{\mathrm{T}} b
$$

\subsection{Determine the number of nearest beacon nodes}

By MLE, $N$, the number of nearest beacon nodes for the blind node, must be determined and divided into groups (three nodes per group because three nodes mean less calculation and high positioning accuracy). ${ }^{(32)}$ The MLE method [described by Eqs. (5)-(7)] is used to calculate the location of the undetermined blind node in each group. Finally, the average value of the raw positioning results in the groups is calculated as the raw positioning result. Through many experiments, we found that $N$ is not large. Therefore, how to determine $N$ becomes the focus of the offline phase. Through experiments, the optimal $N$ is determined to be four for this system. 


\subsection{Improved weighted centroid localization algorithm}

It was found through experiments that the degree of matching between the lognormal distribution model and the actual situation decreases with increasing distance. In view of this phenomenon, the IWCLA based on MLE is proposed in this paper to revise the raw positioning result, as shown in Fig. 2.

Point X1 is the blind node given by Eq. (7). Point X2 is the revised node. Their coordinates are $\left(x_{1}, y_{1}\right)$ and $\left(x_{2}, y_{2}\right)$, respectively. Point $\mathrm{C} 1$ is a beacon node and $\mathrm{cl}$ is the circle centered at point $\mathrm{C} 1$. Point $\mathrm{B} 1$ is the intersection between line $\mathrm{C} 1 \mathrm{X} 1$ and circle $\mathrm{cl}$; its coordinates are $\left(X_{B_{1}}, Y_{B_{1}}\right)$. As the accuracy of the model decreases with increasing distance, a greater weight is given to the near point. Similarly, points B2 and B3 are obtained by the previous method. The IWCLA is shown as

$$
\begin{aligned}
& x_{2}=\frac{r_{2}^{n}+r_{3}^{n}}{2\left(r_{1}^{n}+r_{2}^{n}+r_{3}^{n}\right)} X_{B_{1}}+\frac{r_{1}^{n}+r_{3}^{n}}{2\left(r_{1}^{n}+r_{2}^{n}+r_{3}^{n}\right)} X_{B_{2}}+\frac{r_{1}^{n}+r_{2}^{n}}{2\left(r_{1}^{n}+r_{2}^{n}+r_{3}^{n}\right)} X_{B_{3}} \\
& y_{2}=\frac{r_{2}^{n}+r_{3}^{n}}{2\left(r_{1}^{n}+r_{2}^{n}+r_{3}^{n}\right)} Y_{B_{1}}+\frac{r_{1}^{n}+r_{3}^{n}}{2\left(r_{1}^{n}+r_{2}^{n}+r_{3}^{n}\right)} Y_{B_{2}}+\frac{r_{1}^{n}+r_{2}^{n}}{2\left(r_{1}^{n}+r_{2}^{n}+r_{3}^{n}\right)} Y_{B_{3}}
\end{aligned}
$$

where $r_{1}, r_{2}$, and $r_{3}$ are the radii of circles c1, c2, and c3, $X_{B_{1}}, X_{B_{2}}$, and $X_{B_{3}}$ are the abscissae of B1, B2, and B3, and $Y_{B_{1}}, Y_{B_{2}}$, and $Y_{B_{3}}$ are the ordinates of B1, B2, and B3, respectively. $n$ is the weighted parameter.

The structure of the radio-propagation-model-based localization algorithm based on the methods proposed above is shown in Fig. 3. First, four nearest-neighbor beacon nodes are selected and their RSSIs are sent to the improved average filters. Secondly, the distances

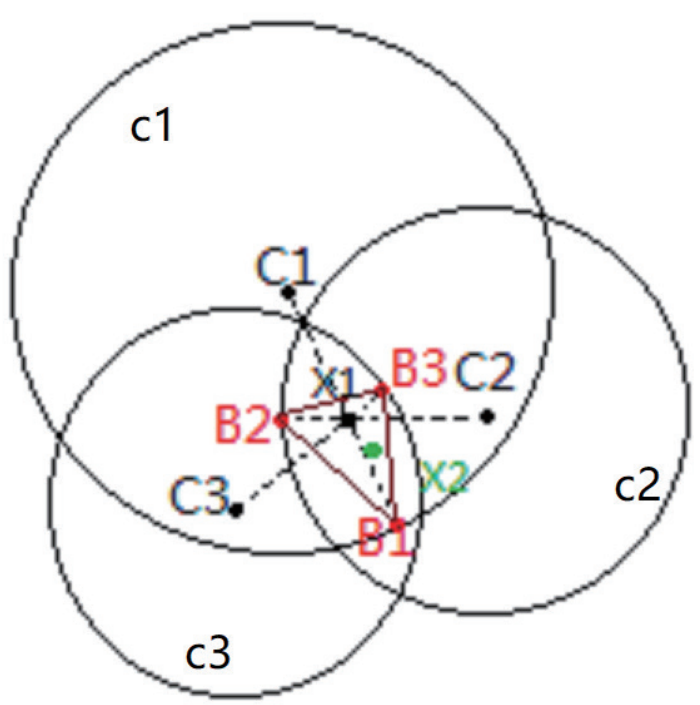

Fig. 2. (Color online) Weighted revision method. 


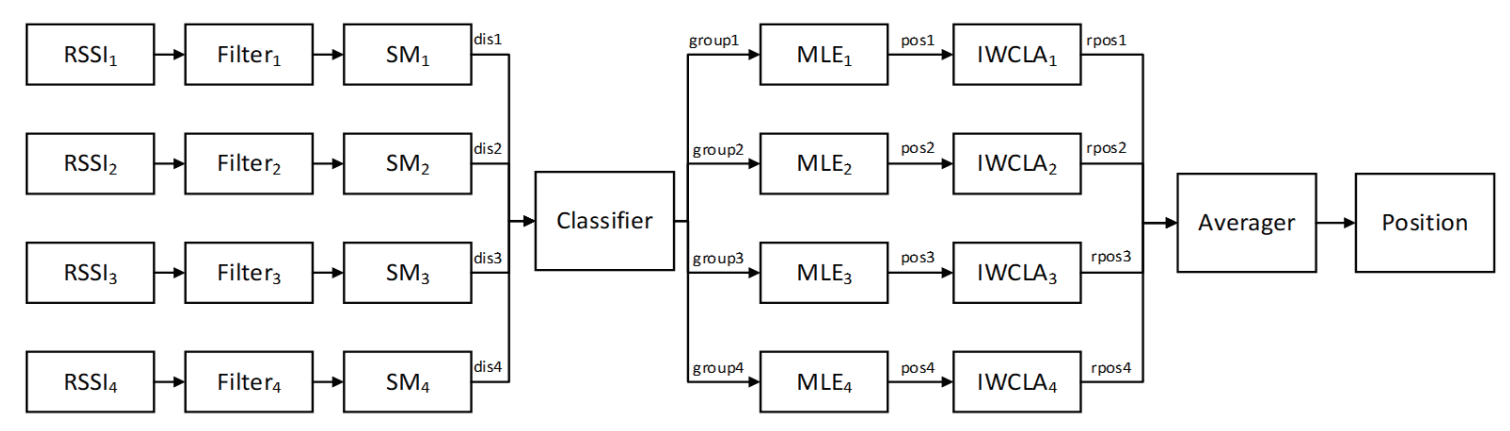

Fig. 3. Structure of the radio-propagation-model-based localization algorithm.

between the blind node and the four nearest beacon nodes are calculated using Eq. (2) (SM) and divided into four groups (three per group) with the position coordinates of the beacon nodes. Then, the four raw positioning results are calculated by MLE and revised using the IWCLA, respectively. Lastly, the four revised positioning results are averaged to obtain the final positioning result.

\section{Improved GRNN Localization Algorithm Based on RSSI}

The radio-propagation-model-based localization algorithm mainly relies on the radio propagation path loss model. However, because of the complex and changeable indoor environment, it is difficult to build a model that fully conforms to the current signal attenuation situation. In contrast, the NN localization algorithm based on the RSSI can achieve better applicability and higher positioning accuracy in the real environment.

Neural network localization algorithms, using the fingerprint database as training data, are based on fingerprint-based positioning technology. Fingerprint-based positioning technology is generally divided into two phases: offline and online phases. The positioning process is shown in Fig. 4.

(1) Offline/training phase

This phase includes data preprocessing and database establishment. In the positioning area, the position of a beacon node is determined at a certain interval, and the RSSI values of all the punctuation marks measured at each beacon node are filtered and stored in the database together with the position coordinate information of the beacon nodes.

(2) Online/positioning phase

In this phase, the signal intensity information detected by the user in real time is compared with that in the fingerprint database, and the target location is calculated using the correlation matching algorithm. Common matching algorithms include the nearest-neighbor method, $\mathrm{K}$ nearest-neighbor method, $\mathrm{K}$ weighted nearest-neighbor method, and NN algorithms.

For NNs, in the training phase, the fingerprint database is used to train the NNs to obtain network parameters that conform to the training data. In the positioning phase, there are two 


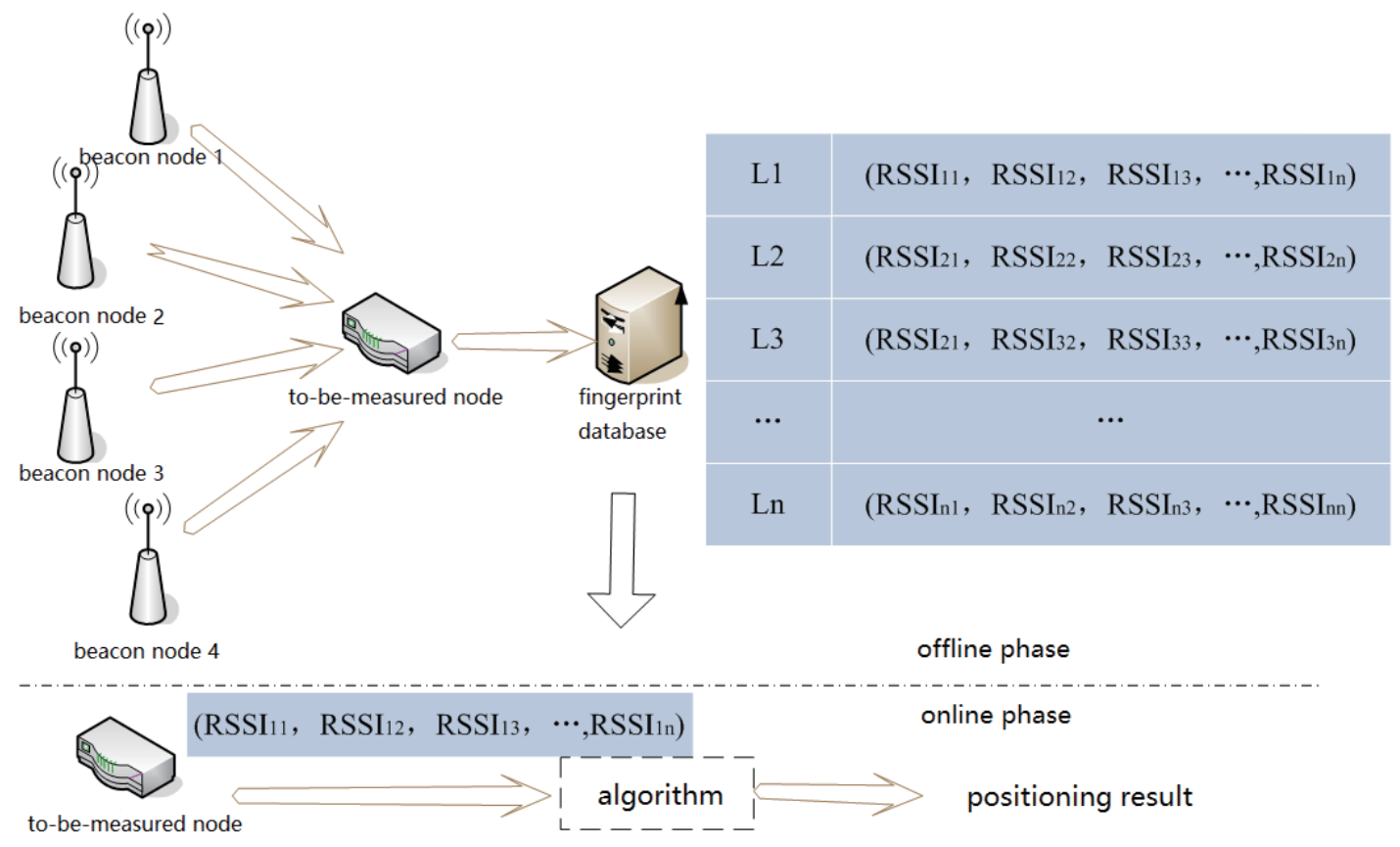

Fig. 4. (Color online) Fingerprint-based positioning flow diagram.

problems that need to be solved: (1) In order to reduce the modeling time and improve the recognition rate of the classification system, an optimal NN structure must be selected for modeling. (2) As the positioning area increases, the amount of beacon nodes and the number of training data also need to be increased. This will result in an increase in NN input, an increase in network dimension, a complex network structure, and a greatly reduced computational speed.

For problem 1, the GRNN is a good choice. Its number of neurons in the hidden layer is the same as the number of input samples. When the number of neurons in the hidden layer is large enough, the network can approximate a function with arbitrary precision. However, with the increase in the number of samples and hidden layer neurons, the network becomes larger and the computational efficiency decreases. This is where the proper dimensionality reduction is needed.

To overcome the shortcomings of the GRNN, an improved GRNN is proposed to solve problem 2. Since the distance of the wireless signal transmission is limited, with the increase in distance, its reliability is reduced. Therefore, instead of using the GRNN directly, the positioning area is divided into several small regions, each of which corresponds to an improved GRNN network, and the classification criterion corresponds to the structure of the nearest beacon nodes for the blind node in the region (for example, if there are six beacon nodes in total, the blind node with the same four nearest-neighbor beacon nodes will be divided into the same region). In this way, the network dimension is reduced, and the positioning accuracy is improved.

On the bases of the structure of the radio-propagation-model-based localization algorithm in Fig. 3, the structure of the improved GRNN is established as shown in Fig. 5. The structure 


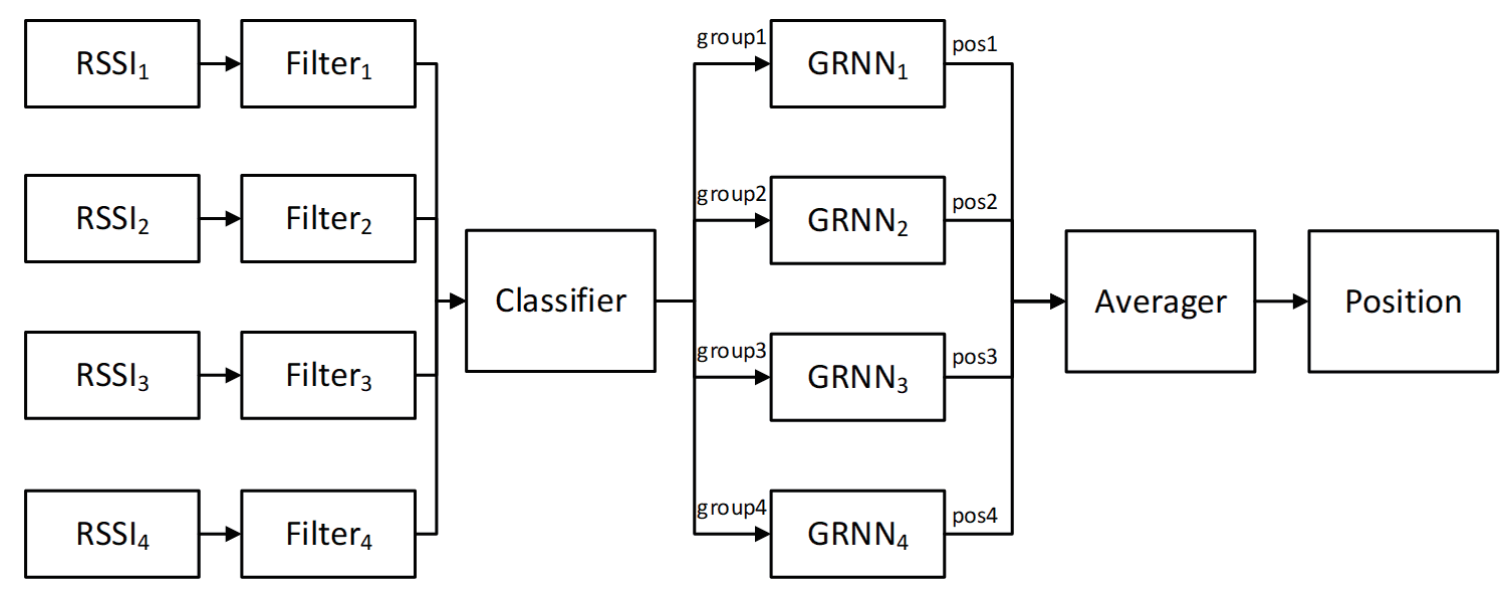

Fig. 5. Structure of the improved GRNN.

is simplified in comparison with the IWCLA, where MLE and the IWCLA are replaced by a single GRNN and the radio-propagation loss model is not needed. Thus, it can avoid possible impacts caused by model inaccuracy as the GRNN is a model-free method. In the proposed improved GRNN method, four single GRNNs are included by setting the number of nearest beacon nodes as four. Every single GRNN has three RSSI inputs and two coordinate outputs of $x$ and $y$. The improved GRNN is proposed to reduce the network dimension and improve the positioning accuracy.

A single GRNN processing phase is shown in Fig. 6. $\operatorname{RSSI}^{(i)}$ and $(x, y)^{(i)}$ mean the $i$ th input and $i$ th output of the $j$ th single $G R N N_{j}$. The maximum values of $i$ and $j$ depend on the size of the dataset and the number of single GRNNs, respectively. An improved GRNN has four single GRNNs and one area may use several improved GRNNs. In the offline phase, each single GRNN corresponds to three particular RSSIs [for example, the three nearest-neighbor beacon nodes for the blind node in the training set are $\mathrm{RSSI}_{1}, \mathrm{RSSI}_{2}$, and $\mathrm{RSSI}_{3}$ with the output coordinates $(x, y)$, which are put in the corresponding single GRNN network for training]. In the online phase, the blind node is estimated in the corresponding area and four corresponding single GRNNs are chosen to form the improved GRNN with other components, as shown in Fig. 5. The three NN algorithms (BPNN, RBFNN, and improved GRNN) are compared in Sect. 6.3, and the improved GRNN model based on the RSSI is verified to be more suitable for an indoor localization system.

\section{Simulation Results}

MATLAB is used to verify the proposed methods and algorithms. The simulation includes comparisons of several filters, and verifications of the efficiency of the IWCLA and improved GRNN. 


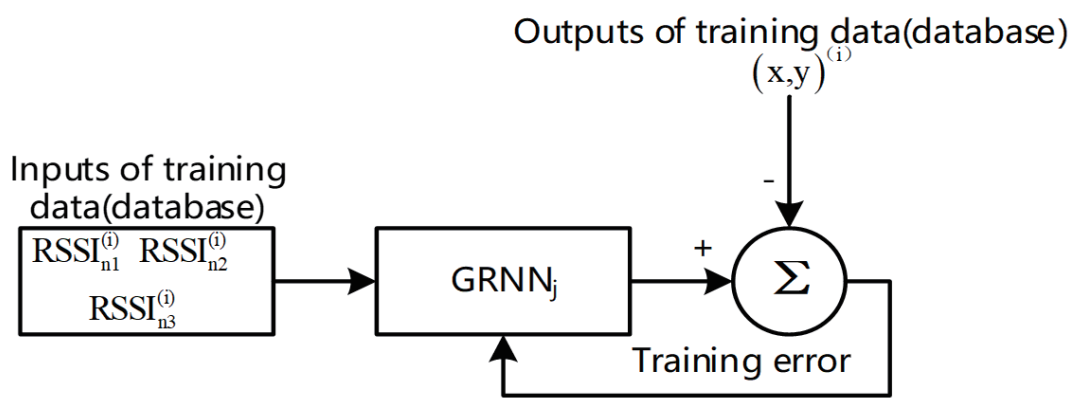

(a)

Real-time RSS

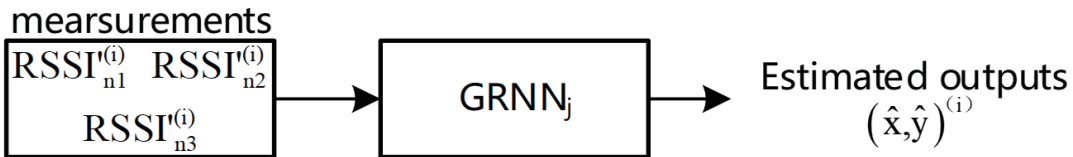

(b)

Fig. 6. (Color online) Single GRNN processing phase. (a) offline and (b) online phases.

\subsection{Noise filtering algorithm}

The signal intensity loss is affected by the reflection of the environment, the multichannel dynamic reflection, and the mutual interference between multiple signal emitters. The loss is referred to as noise. The measurement statistics indicate that the noise is a Gaussian distribution function with an average of zero.

Here, when the MATLAB normrnd (MU, SIGMA) function is used to simulate environmental noise, the Gaussian distribution function parameter MU represents the average and SIGMA represents the standard deviation. Normrnd $(0,7)$ is used to simulate noise in the experiment. We assume that the actual signal strength is $-60 \mathrm{dBm}$ and noise interference is added to it with the sampling period of $1 \mathrm{~s}$.

In addition to the two average filters, there is also a feedback filter ${ }^{(30)}$ to reduce positioning delay and a smoothing filter ${ }^{(32)}$ to make the positioning data more reliable. In the simulation, the four filters are each verified. The simulation results are shown in Fig. 7.

In Fig. 7, the orange solid line represents the raw signal without noise. The green dashed line represents the raw signal with noise. The dashed lines of other colors represent the signals filtered by different filters. The mean-square-root errors of the average filtered, feedback filtered, smooth filtered, improved average filtered, and raw signals are 0.18, 0.41, 0.25, 0.16, and $0.67 \mathrm{dBm}$, respectively. Although the filtering effects of the average and smooth filters are not as good as that of the improved average filter, their values are also close to that of the raw signal without noise, indicating that they have better filtering effects than the feedback filter. The signals filtered by the average, feedback, and improved average filters gradually level off with time. Although the feedback filter reduces noise to a certain extent, it does not exhibit a good filtering effect, and the filtered RSSI value still changes with noise fluctuation. It is obvious that the improved average filter has the best filtering effect among the four filters. 


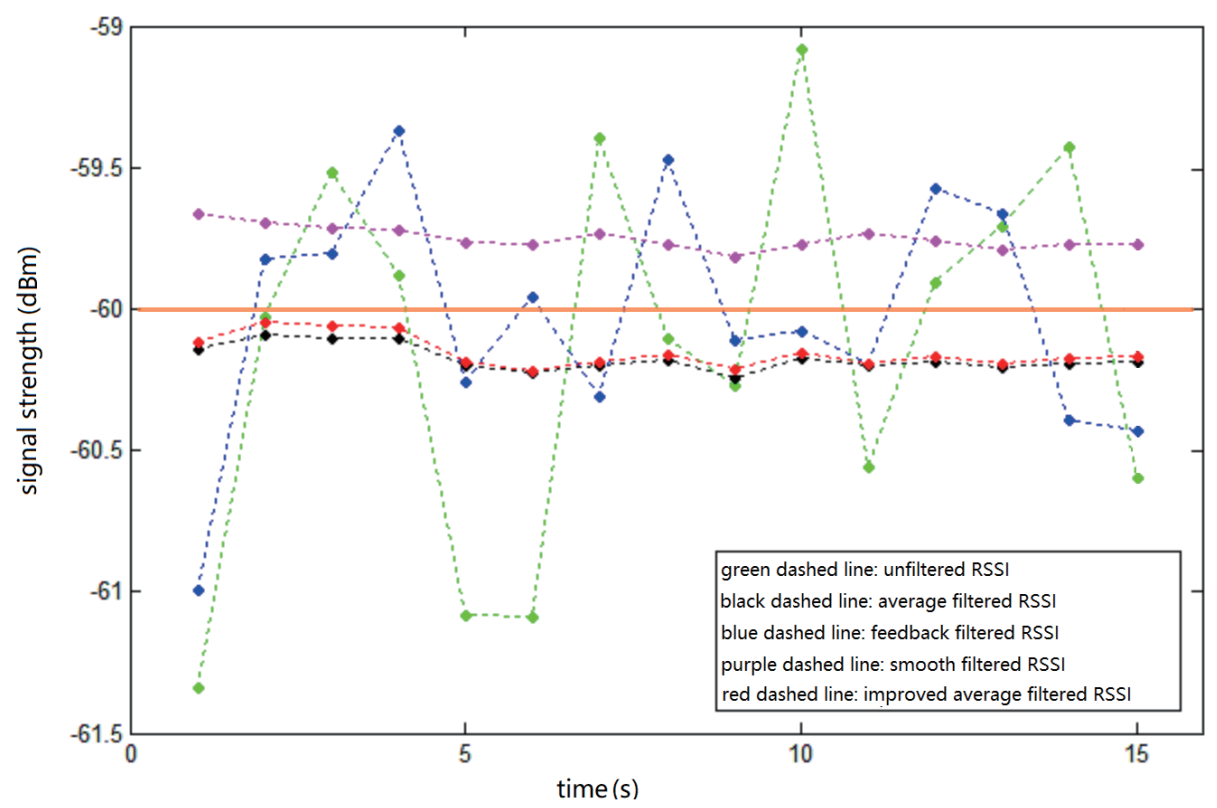

Fig. 7. (Color online) Simulation results of noise filtering algorithm.

\subsection{Improved weighted centroid localization algorithm}

The static positioning simulation scene described in this section is shown in Fig. 8. The positioning area is $40 \times 40 \mathrm{~m}^{2}$. Seven beacon points are set in the area, and their coordinates are $(10,0),(30,0),(0,20),(20,20),(40,20),(10,40)$, and $(30,40)$. In this scenario, the lognormal distribution model is used in Eq. (2), where the path loss coefficient is set to three, and the noise is set to a Gaussian distribution random number with a mean of zero and a standard deviation of seven.

In the simulation, 100 points to be located are randomly generated, and the number of their nearest beacon nodes, $N$, is waiting to be determined. $C_{N}^{3}$ groups are calculated by MLE [shown in Eqs. (5)-(7)] and the average result is the final positioning result. With different $N$ values, different positioning results are obtained as shown in Fig. 8.

According to Eq. (6), the greater the number $n$, the higher the accuracy of the positioning, and the higher the cost of calculation and hardware. In practical applications, the distant nodes are unreliable because of interference effects, thus causing great error in the positioning. The grouping method is used when $N$ nearest-neighbor beacon nodes are selected and divided into $C_{N}^{3}$ groups to use the MLE in line with the rule of three nodes per group. ${ }^{(32)}$ We compare the positioning result with different $N$ values, as shown in Fig. 8 (the blue asterisk represents the beacon point, the black asterisk represents the target positioning point, and the red circle represents the actual positioning point). The calculated average distance errors are 2.387, 1.015, 2.272 , and $5.642 \mathrm{~m}$ with $N=3,4,5$, and 6 , respectively. It can be clearly seen in the figure that the distance between the target and the actual positioning point is the smallest when $N=4$. According to the simulation results, $N=4$ is selected for the best positioning effect. 


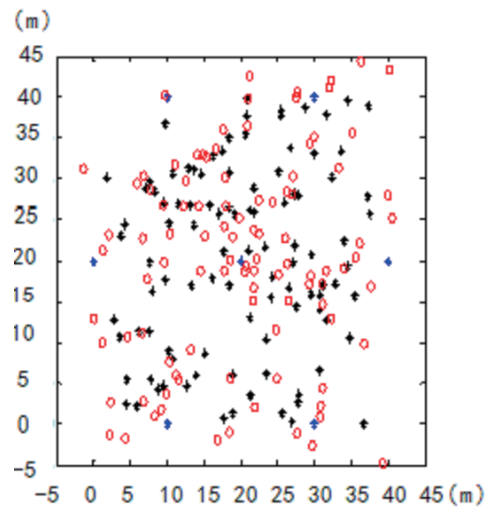

(a)

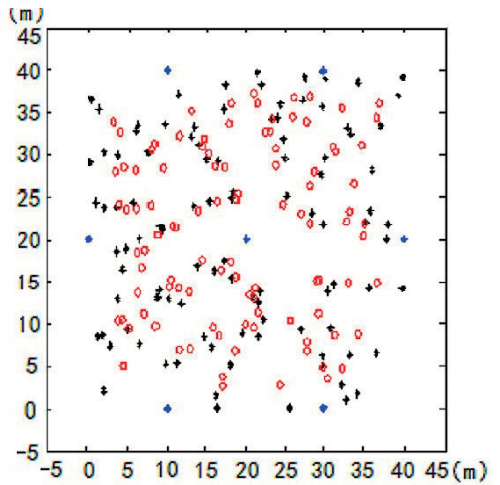

(c)

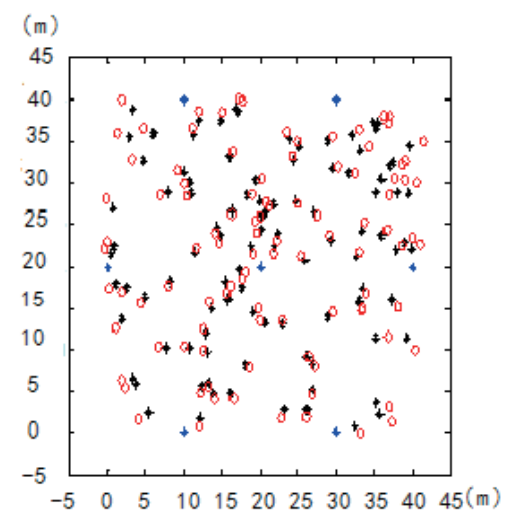

(b)

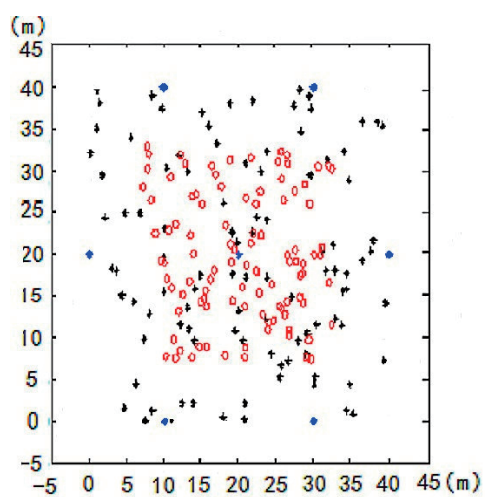

(d)

Fig. 8. (Color online) Simulation results of the MLE method. (a) $N=3$, (b) $N=4$, (c) $N=5$, and (d) $N=6$.

However, it is found through experiments that the degree of matching between the lognormal distribution model and the actual situation decreases with increasing distance. When the IWCLA presented in Sect. 4.3 is used to revise the positioning result based on the MLE with $N=4$, the average distance error is reduced to $1.0 \mathrm{~m}$. The simulation result is shown in Fig. 9.

\subsection{Comparison of three NNs}

This simulation is set up under the MATLAB simulation environment. Six beacon points are arranged in an $80 \times 60 \mathrm{~m}^{2}$ rectangular positioning area and their coordinates are $(20,20)$, $(40,20),(60,20),(20,40),(40,40)$, and $(60,40)$. Then, 500 points are generated randomly and their RSSI values are calculated from the six beacon nodes in accordance with the lognormal distribution model (in the real experiment, an RSSI value can be measured directly using reliable hardware). Then, the coordinates of the 500 points and $500 \times 6$ RSSI values are stored in the file as the training data of the NN. Twenty points to be measured are randomly generated, and the RSSI values they receive is used as the input of the NN. Here, the path-loss coefficient of the lognormal distribution model is set to three, and the noise in the positioning scene is set to a Gaussian distribution random number with a mean of zero and a standard deviation of seven. 


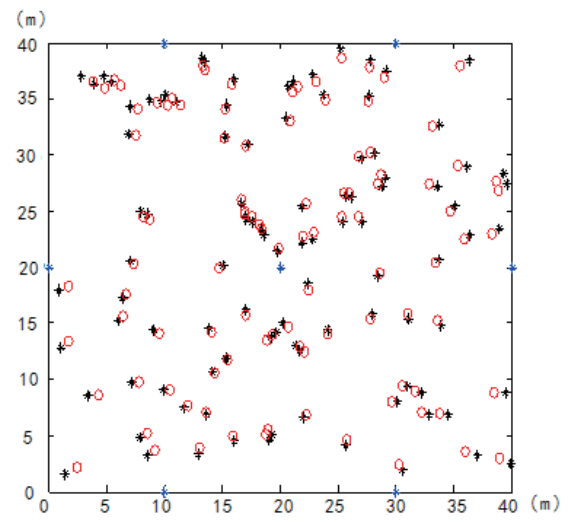

Fig. 9. (Color online) Simulation results of the IWCLA.

The BPNN and RBFNN have the same input and output layers. Each point to be measured receives RSSIs from six different beacon nodes, and the coordinates of each point are needed. Therefore, the number of neurons in the input layer is set to six corresponding to six beacon nodes, and the number of neurons in the output layer is set to two corresponding to the coordinates of $x$ and $y$. The positioning area is divided into several regions, each corresponding to an improved GRNN with four inputs and two outputs.

These three NNs have different structures and hidden layers. On the basis of the experimental results, the BPNN's hidden layer is set to one with three neurons, the RBFNN's hidden layer is set to one with six neurons, and the single GRNN's (an improved GRNN has four single GRNNs) hidden layer is set to one with $n_{p}$ (the number of input samples in the $p$ th single GRNN) neurons.

Figures 10(a)-10(c) show the simulation results of the BPNN, RBFNN, and improved GRNN, respectively. Cyan asterisks indicate the beacon nodes, the black asterisk is the actual position of the point, that is, the expected value of NNs, and the small red circle is the position calculated by NNs. In order to adopt the best NN results in the positioning system of the WSN, the BPNN, RBFNN, and improved GRNN models are compared and analyzed from the three aspects of training time, the number of neurons in the hidden layer, and the average distance error.

Table 1 shows the training time, the number of neurons in the hidden layer, and average distance errors of three different NNs. The learning rate of the BPNN is obviously lower than those of the other two networks. According to the Euclidean distance calculation, the average distance error of the improved GRNN is less than those of the other two networks. From the perspective of training time and average distance error, the improved GRNN network is obviously superior to the other two.

\section{Experiment Results}

The positioning system is composed of ZigBee gateway and ZigBee beacon nodes. The Mdv-stm32-107 and emb-stm32w-m1 development boards are respectively used in the designs. 


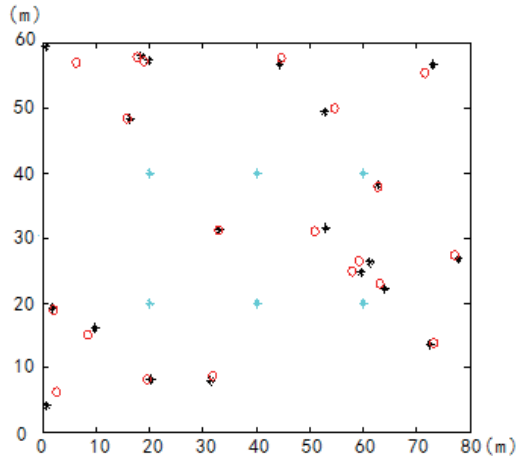

(a)

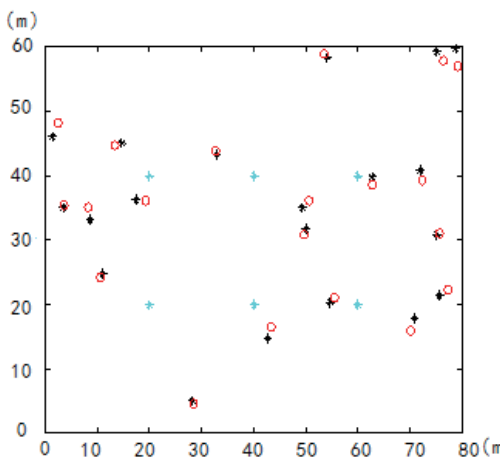

(b)

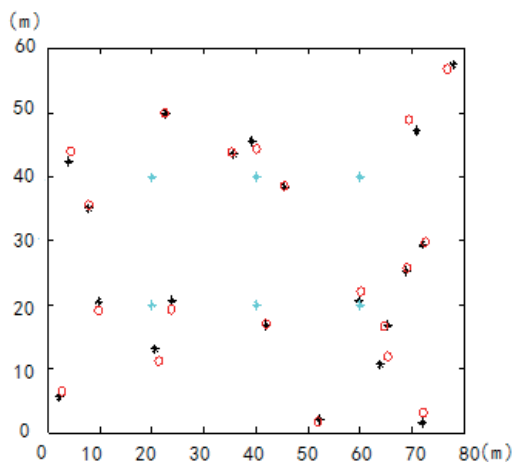

(c)

Fig. 10. (Color online) Simulation results of three NNs. (a) BPNN, (b) RBFNN, and (c) improved GRNN.

Table 1

Performance of three NNs.

\begin{tabular}{lccc}
\hline Type of NN & Training time (s) & No. of neurons in hidden layer & Average distance error (m) \\
\hline BPNN & 19.24 & 3 & 1.46 \\
RBFNN & 7.92 & 6 & 1.43 \\
Improved GRNN & 0.13 & $n_{p}$ in $p$ th single GRNN & 1.08 \\
\hline
\end{tabular}

The MDV-STM32-107 development board is used for the ZigBee gateway to be placed on the moving objects to be located. The system is also used for networking. Beacon frames containing RSSI are sent, positioning calculation is performed, and positioning results are sent out.

The EMB-STM32W-M1 development board is fixed indoors, used for the ZigBee beacon node, and used to join the ZigBee network when the system is in operation. It receives RSSIs and forward them to the gateway.

We place the gateway on top of the car and the beacon nodes at a fixed position of the garage at the same height as the gateway. Since the road in the garage is flat, the vertical position of the gateway will not change when the car is being driven in to the garage. Thus, although the experiment is conducted in a 3D environment, a 2D positioning method can still be used.

The experiment is carried out in a parking lot with a size of $30 \times 60 \mathrm{~m}^{2}$. Nineteen beacon nodes between carports are relatively evenly distributed in the parking lot, as shown in Fig. 11 (the black asterisk represents the target position; the red circle represents the actual position in experiment 1; the blue circle represents the actual position in experiment 2). The radiopropagation-model-based and improved GRNN localization algorithms are each used to locate mobile nodes.

(1) Experiment 1: Radio-propagation-model-based localization algorithm

Before the experiment, preparations should be made; that is, in the experiment scene, mobile nodes should be placed at distances of $0.5,1,1.5,2,3,4,5,6$, and $7 \mathrm{~m}$ from any beacon node. The RSSI values accepted by mobile nodes should be measured many times, and the average value for the same position should be calculated. Then, Eq. (2) is used to fit the RSSI and Euclidean distance d, that is, A and B are calculated as -60.54 and -29.96 , respectively. 


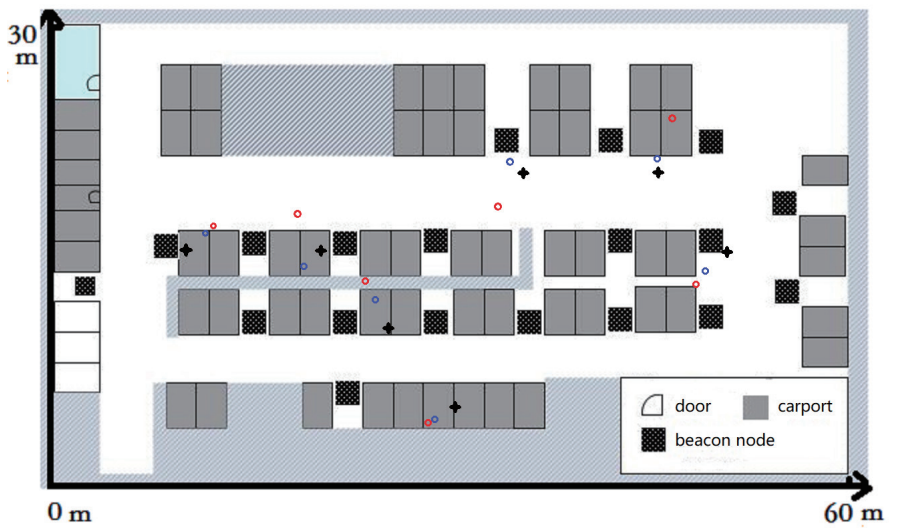

Fig. 11. (Color online) Layout of parking lot test scene.

Table 2

Results of the two algorithms.

\begin{tabular}{lccccc}
\hline \multirow{2}{*}{ No. } & \multirow{2}{*}{ Target position } & \multicolumn{2}{c}{ Experiment 1 } & \multicolumn{2}{c}{ Experiment 2 } \\
\cline { 3 - 6 } & & Actual position & Positioning error $(\mathrm{m})$ & Actual position & Positioning error (m) \\
\hline 1 & $(20,15)$ & $(18.1,17.5)$ & 3.1 & $(18.6,14.1)$ & 1.6 \\
2 & $(25,10)$ & $(23.1,13.2)$ & 3.7 & $(23.9,12.1)$ & 2.3 \\
3 & $(10,15)$ & $(12.1,16.5)$ & 2.5 & $(11.8,16.3)$ & 2.2 \\
4 & $(30,5)$ & $(27.7,3.9)$ & 2.5 & $(28.3,4.1)$ & 1.9 \\
5 & $(35,20)$ & $(32.9,17.9)$ & 2.9 & $(33.8,20.9)$ & 1.5 \\
6 & $(50,15)$ & $(47.5,12.8)$ & 3.3 & $(48.2,13.9)$ & 2.1 \\
7 & $(45,20)$ & $(45.9,23.8)$ & 3.9 & $(44.7,21.1)$ & 1.1 \\
\hline
\end{tabular}

(2) Experiment 2: Improved GRNN localization algorithm

Before the experiment, the training data must be collected, and the position of the carport is selected as the training points (10 training points per carport). The $62 \times 10$ data values collected are used to train the improved GRNN.

The results of the experiment are shown in Table 2. The positioning accuracies in experiments 1 and 2 are 2.5-3.9 m (mean: $3.1 \mathrm{~m}$ ) and 1.1-2.3 m (mean: $1.8 \mathrm{~m}$ ), respectively. The accuracy of the improved GRNN localization algorithm is significantly higher than that of the radio-propagation-model-based localization algorithm in the real environment. Since the positioning area is large and the garage is full of vehicles that may cause disturbances or occlusions to the positioning, it is still a challenge to locate the vehicle in the garage with good positioning accuracy and positioning stability. In contrast to other positioning methods used in the garage, our methods consider both the positioning accuracy and positioning stability (based on the WSN and RSSI), and yield a good positioning result.

\section{Conclusions}

We studied the indoor wireless positioning technology based on the RSSI and GRNN. Firstly, an improved average filter was proposed. Secondly, an improved weighted centroid algorithm was proposed to revise the approximate position of the mobile node by MLE. Then, 
an improved GRNN was proposed in response to the GRNN's deficiency in achieving better applicability and higher positioning accuracy in the real environment. Finally, the effectiveness of the proposed methods was verified in different cases through simulations and experiments.

\section{Acknowledgments}

This work was supported in part by the National Natural Science Foundation of China (61733013, u1764261, 61773289), the Natural Science Foundation of Shanghai (19ZR1461400, 17ZR1445800), and Fundamental Research Funds for the Central Universities.

\section{References}

1 P. Neirotti, A. De Marco, A. C. Cagliano, G. Mangano, and F. Scorrano: Cities 38 (2014) 25. https://doi. org/10.1016/j.cities.2013.12.010

2 J. Jin, J. Gubbi, S. Marusic, and M. Palaniswami: IEEE Int. Things J. 1 (2014) 112. https://doi.org/10.1109/ JIOT.2013.2296516

3 J. M. Nocquet: Tectonophysics 579 (2012) 220. https://doi.org/10.1016/j.tecto.2012.03.037

4 K. Yu, C. Rizos, D. Burrage, A. G. Dempster, K. Zhang, and M. Markgraf: EURASIP J. Adv. Signal Process 2014 (2014) 134. https://doi.org/10.1186/1687-6180-2014-134

5 C. N. Huang and C. T. Chan: Procedia Comput. Sci. 5 (2011) 58. https://doi.org/10.1016/j.procs.2011.07.010

6 Q. Xu, X. Li, and C. Y. Chan: Sensors 17 (2017) 1431. https://doi.org/10.3390/s17061431

7 X. Qu, B. Soheilian, and N. Paparoditis: Intelligent Vehicles Symp. (IEEE, 2015) 605. https://doi.org/10.1109/ IVS.2015.7225751

8 J. Wagner, C. Isert, A. Purschwitz, and A. Kistner: Int. Conf. Indoor Positioning and Indoor Navigation (IEEE, 2010) 1. https://doi.org/10.1109/IPIN.2010.5646710

9 A. Ibisch, S. Houben, M. Schlipsing, R. Kesten, P. Reimche, F. Schuller, and H. Altinger: IEEE Intelligent Vehicles Symp. Proc. (IEEE, 2014) 426. https://doi.org/10.1109/IVS.2014.6856567

10 S. Vasuhi and V. Vaidehi: Inf. Fusion 27 (2016) 41. https://doi.org/10.1016/j.inffus.2015.05.004

11 J. M. Pak, C. K. Ahn, P. Shi, Y. S. Shmaliy, and M. T. Lim: IEEE Trans. Ind. Electron. 64 (2017) 5182. https:// doi.org/10.1109/TIE.2016.2608897

12 H. Lin, L. Chen, H. Lin, L. Chen, H. Lin, and L. Chen: Int. Conf. Information Networking (IEEE, 2016$) 291$. https://doi.org/10.1109/ICOIN.2016.7427079

13 C. Chen, C. Yan, H. Yi, H. Q. Lai, Z. Feng, and K. J. R. Liu: IEEE Internet Things J. 4 (2017) 122. https://doi. org/10.1109/JIOT.2016.2628713

14 Y. Zhuang, J. Yang, Y. Li, L. Qi, and N. El-Sheimy: Sensors 16 (2016) 596. https://doi.org/10.3390/s16050596

15 S. H. Fang, C. H. Wang, T. Y. Huang, C. H. Yang, and Y. S. Chen: IEEE Commun. Lett. 16 (2012) 564. https:// doi.org/10.1109/LCOMM.2012.022112.120131

16 H. Laitinen, J. Lahteenmaki, and T. Nordstrom: IEEE VTS 53rd Vehicular Technology Conf., Spring 2001 Proc. (IEEE, 2001) 2504. https://doi.org/10.1109/VETECS.2001.944052

17 A. H. Ismail, H. Kitagawa, R. Tasaki, and K. Terashima: IEEE Int. Conf. Systems, Man, and Cybernetics (IEEE, 2017) 9. https://doi.org/10.1109/SMC.2016.7844461

18 M. Zhou, F. Qiu, K. Xu, Z. Tian, and H. Wu: Comput. Commun. 86 (2016) 57. https://doi.org/10.1016/ j.comcom.2016.03.020

19 L. W. Yeh, M. S. Hsu, Y. F. Lee, and Y. C. Tseng: Sensors (IEEE, 2010) 1463. https://doi.org/10.1109/ ICSENS.2009.5398451

20 H. Lemelson, S. Schnaufer, and W. Effelsberg: IEEE Int. Conf. Pervasive Computing and Communications Workshops (IEEE, 2010) 540. https://doi.org/10.1109/PERCOMW.2010.5470497

21 S. P. Kuo and Y. C. Tseng: IEEE Trans. Knowl. Data Eng. 20 (2008) 678. https://doi.org/10.1109/ TKDE.2007.190730

22 C. H. Cheng, W. J. Luo, Y. W. Lin, and C. C. Sun: IEEE Int. Symp. Consumer Electronics (IEEE, 2013$) 73$. https://doi.org/10.1109/ISCE.2013.6570259

23 R. J. Kuo, M. C. Shieh, J. W. Zhang, and K. Y. Chen: Rob. Computer-Integr. Manuf. 29 (2013) 431. https://doi. org/10.1016/j.rcim.2013.04.002 
24 B. L. Hu, J. R. Xu, H. H. Gao, J. H. Liu, and K. R. Wang: 2013 Int. Conf. Optoelectronics and Microelectronics (ICOM) (IEEE, 2013) 2675. https://doi.org/10.4028/www.scientific.net/AMM.423-426.2675

25 H. L. Ding, W. W. Y. Ng, P. P. K. Chan, D. L. Wu, X. L. Chen, and D. S. Yeung: Int. Conf. Machine Learning \& Cybernetics (IEEE, 2010) 1147. https://doi.org/10.1109/ICMLC.2010.5580925

26 D. Guo, Y. Zhang, Q. Xiang, and Z. Li: Math. Prob. Eng. 2014 (2014) 9. https://doi.org/10.1155/2014/420482.

27 M. S. Rahman: Arab. J. Sci. Eng. 37 (2012) 1043. https://doi.org/10.1007/s13369-012-0218-1

28 V. Abhayawardhana, I. Wassell, D. Crosby, M. Sellars, and M. Brown: 2005 IEEE 61st Vehicular Technology Conf. (2005) 73. https://doi.org/10.1109/VETECS.2005.1543252

29 N. B. Priyantha, A. Chakraborty and H. Balakrishnan: Proc. 6th Annual Int. Conf. Mobile Computing and Networking (ACM, 2000) 32. https://doi.org/10.1145/345910.345917

30 S. J. Halder, T. Y. Choi, H. P. Jin, S. H. Kang, S. W. Park, and J. G. Park: Tenth Int. Conf. Information Integration and Web-based Applications Services (DBLP, 2008) 367. https://doi.org/10.1145/1497308.1497374

31 W. Ren, L. Xu, D. Zou, and Z. Deng: J. Data Acquisition 24 (2009) 757. https://en.cnki.com.cn/Article_en/ CJFDTOTAL-SJCJ200906012.htm

32 Q. Yang: Research on Fingerprint-based Wireless Indoor Location Algorithm, Zhejiang University (2011) (Thesis in Chinese). https://dwz.cn/51RfGjzO

\section{About the Authors}

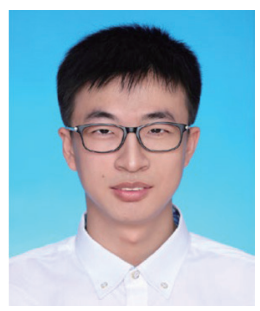

Shuqi Xu received his B.Sc. degree in electronic information engineering from Jiangsu University, Jiangsu, China, in 2018. He is currently pursuing a $\mathrm{Ph}$.D. degree in control theory and control engineering at Tongji University, Shanghai, China. His current research interests include the dexterous manipulation of robotic arms and intelligent robots.

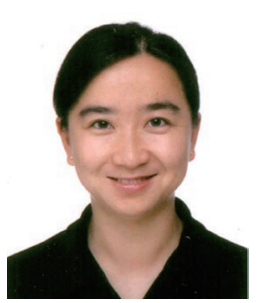

Zhuping Wang received her B.Eng. and M.Eng. degrees from the Department of Automatic Control, Northwestern Polytechnic University, China, in 1994 and 1997, respectively, and her Ph.D. degree from the National University of Singapore, Singapore, in 2003. She is currently a professor with the College of Electronics and Information Engineering, Tongji University, Shanghai, China. Her research interests include the intelligent control of robotic systems and nonholonomic control systems.

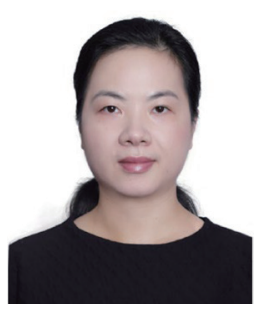

Hao Zhang received her B.Sc. degree in automatic control from the Wuhan University of Technology, Wuhan, China, in 2001, and her Ph.D. degree in control theory and control engineering from the Huazhong University of Science and Technology, Wuhan, in 2007. She is currently a professor at the School of Electronics and Information Engineering, Tongji University, Shanghai, China. Her current research interests include network-based control systems, multiagent systems, and complex networks. 
Shuzhi Sam Ge received his B.Sc. degree in control engineering from the Beijing University of Aeronautics and Astronautics, Beijing, China, in 1986 and his Ph.D. degree in mechanical/electrical engineering from the Imperial College London, London, U.K., in 1993. He is the Director of Social Robotics Laboratory, Smart Systems Institute and a professor in the Department of Electrical and Computer Engineering, National University of Singapore, Singapore. He has coauthored seven books and more than 300 international journal and conference papers. His current research interests include social robotics, adaptive control, intelligent systems, and artificial intelligence. 